\title{
Hippocampal CA3 NMDA Receptors Are Crucial for Adaptive Timing of Trace Eyeblink Conditioned Response
}

\author{
Yasushi Kishimoto, ${ }^{1,2,3}$ Kazu Nakazawa, ${ }^{3,4}$ Susumu Tonegawa, ${ }^{4}$ Yutaka Kirino, ${ }^{2}$ and Masanobu Kano ${ }^{1}$ \\ ${ }^{1}$ Department of Cellular Neurophysiology, Graduate School of Medical Science, Kanazawa University, Kanazawa 920-8640, Japan, ${ }^{2}$ Laboratory of \\ Neurobiophysics, School of Pharmaceutical Sciences, The University of Tokyo, Tokyo 113-0003, Japan, ${ }^{3}$ National Institute of Mental Health, National \\ Institutes of Health, Bethesda, Maryland 20892-4405, and ${ }^{4}$ Howard Hughes Medical Institute, The Picower Center for Learning and Memory, \\ RIKEN-Massachusetts Institute of Technology Neuroscience Research Center, Center for Cancer Research, and Departments of Biology and Brain and \\ Cognitive Sciences, Massachusetts Institute of Technology, Cambridge, Massachusetts 02139-4307
}

Classical conditioning of the eyeblink reflex is a simple form of associative learning for motor responses. To examine the involvement of hippocampal CA3 NMDA receptors (NRs) in nonspatial associative memory, mice lacking an NR1 subunit selectively in adult CA3 pyramidal cells [CA3-NR1 knock-out (KO) mice] were subjected to eyeblink conditioning paradigms. Mice received paired presentations of an auditory conditioned stimulus (CS) and a periorbital shock unconditioned stimulus (US). With repeated presentation of the CS followed by the US, wild-type mice learned to blink in anticipation of the US before its onset. We first confirmed that wild-type mice require an intact hippocampus in the trace version of eyeblink conditioning in which the CS and US do not overlap, creating a stimulusfree time gap of $500 \mathrm{~ms}$. Under the same condition, CA3-NR1 KO mice successfully acquired conditioned responses (CRs) during the $10 \mathrm{~d}$ acquisition sessions, whereas the extinction of CRs was impaired on the first day of extinction sessions. Importantly, CA3-NR1 KO mice were impaired in the formation of an adaptively timed CR during the first five trials in the daily acquisition sessions. The aberrantly timed $\mathrm{CR}$ was also observed in the extinction sessions in accordance with the impaired extinction of CRs. These results indicate that CA3-NR1 KO mice are unable to rapidly retrieve adaptive CR timing, suggesting that CA3 NRs play a crucial role in the memory of adaptive CR timing in trace conditioning.

Key words: NMDA receptor; hippocampus; area CA3; eyeblink conditioning; nonspatial associative memory; mouse

\section{Introduction}

Classical conditioning of the eyeblink reflex has been a useful behavioral model for investigating the neural substrate underlying learning and memory (for review, see Thompson and Kim, 1996; Thompson et al., 1997; Yeo and Hesslow, 1998; McEchron and Disterhoft, 1999). After repeated presentation of a tone [conditioned stimulus (CS)] followed by a periorbital shock [unconditioned stimulus (US)], the subject blinks in anticipation of the US, and this anticipatory response is called the conditioned response (CR). In the delay version of eyeblink conditioning, a tone CS precedes, overlaps, and coterminates with an aversive US, whereas in trace conditioning, the CS and US do not overlap, creating a stimulus-free time gap (i.e., trace interval) (Gormezano et al., 1987). Accumulating evidence suggests that in trace conditioning, but not in delay conditioning, the hippocam-

Received Sept. 28, 2005; revised Dec. 11, 2005; accepted Dec. 11, 2005

This work was supported in part by Grants-in-Aid for Scientific Research 15.8589 (Y. Kishimoto), 17023021 (M.K.), and 17100004 (M.K.) from the Ministry of Education, Culture, Sports, Science and Technology of Japan and by the National Institutes of Health-National Institute of Mental Health Intramural Program. Y. Kishimoto is the recipient of a fellowship from the Japan Society for the Promotion of Science and Japan-U.S. Brain Research Cooperation Program. We thank K. Hashimoto for preparing this manuscript.

Correspondence should be addressed to Dr. Masanobu Kano, Department of Cellular Neuroscience, Graduate School of Medicine, Osaka University, 2-2 Yamada-oka, Suita, 0saka 565-0871, Japan. E-mail: mkano@ cns.med.osaka-u.ac.jp.

DOl:10.1523/JNEUROSCI.4142-05.2006

Copyright $\odot 2006$ Society for Neuroscience $\quad$ 0270-6474/06/261562-09\$15.00/0 pus is required for acquisition of adaptively timed CR in anticipation of the US (Port et al., 1986; Solomon et al., 1986; James et al., 1987; Moyer et al., 1990). Systemic infusion of NMDA receptor (NR) antagonists and global genetic ablation of an NR subunit $\epsilon 1$ (NR2A) impaired acquisition and timing of trace eyeblink conditioning (Thompson and Disterhoft, 1997; Kishimoto et al., 2001; Takatsuki et al., 2001).

In the CA3 region of the hippocampus, the dynamics of the extensive recurrent network mediated by massive longitudinal association fibers has long been suggested to play a dominant role in certain types of learning and memory such as auto-associative recall (Marr, 1971; Morris and McNaughton, 1987; Hasselmo et al., 1995, 2002; Hasselmo and McClelland, 1999; Lisman, 1999; Kesner et al., 2000). Several models have pointed out that the hippocampal CA3 region is involved in making predictions in sequence learning (Abbott and Blum, 1996; Jensen and Lisman, 1996; Levy, 1996; August and Levy, 1999). In particular, Rodriguez and Levy (2001) have proposed, based on temporally asymmetric synaptic modification (Levy and Steward, 1983), that CA3 network functions as a time-indexed encoding device for the CS in trace conditioning.

We recently generated genetically engineered mice in which a gene for the NR1 subunit of NRs is ablated only in adult hippocampal CA3 pyramidal cells [referred to as CA3-NR1 knockout (KO) mice] (Nakazawa et al., 2002). CA3-NR1 KO mice were 
unable to fully recall the memory when recalling cues were degraded (Nakazawa et al., 2002, 2004), supporting that CA3 NRs play an important role in recall of spatial reference memory by pattern completion (Marr, 1971). Furthermore, recent studies using CA3-NR1 KO mice have suggested that CA3 NRs play a crucial role in rapid hippocampal encoding of a novel information for fast learning of a one-time experience (Nakazawa et al., 2003, 2004).

The availability of the CA3-NR1 KO mice provided an opportunity to test the hypothesis that the recurrent CA3 network with modifiable synaptic strength supports acquisition of well timed CRs in trace conditioning. Our results indicate that CA3-NR1 $\mathrm{KO}$ mice were unable to rapidly retrieve adaptive CR timing during the trace conditioning and were impaired in its extinction.

\section{Materials and Methods \\ Animals}

CA3-NR1 KO mice (mutant) and their homozygously floxed-NR1 littermates (control) were obtained as described previously (Nakazawa et al., 2002). In this mutant, NR1 mRNA was almost completely abolished from CA3 pyramidal cells of both dorsal and ventral areas of CA3 by 18 weeks of age. Accordingly, no synaptically evoked NMDA channel currents were detected from CA3 pyramidal cells by whole-cell patch recording while the NR currents from CA1 pyramidal cells and dentate granule cells were intact. We used male mice, $18-26$ weeks of age, at the time of surgery. After behavioral analysis, genotype was confirmed by PCR of genomic DNA extracted from the tail of each mouse. For the lesion experiment, we purchased C57BL/6 strains of wild-type mice from Japan SLC (Hamamatsu, Japan). All subjects were maintained on a $12 \mathrm{~h}$ light/ dark cycle with food and water available ad libitum. During the course of the present study, the care of the animals conformed to the guidelines established by the University Institutional Animal Investigation Committee.

\section{Hippocampal lesion procedure}

Bilateral lesions of the hippocampus were made according to the procedure described previously (Cho et al., 1999). C57BL/6 mice at the age of 16 weeks were anesthetized by ketamine $(80 \mathrm{mg} / \mathrm{kg}$, i.p. $)$ and xylazine $(20$ $\mathrm{mg} / \mathrm{kg}$, i.p.) and were placed in a stereotaxic apparatus (Narishige, Tokyo, Japan). The hippocampal lesioned (HIP) group received 24 injections of ibotenic acid ( $~ 0.06-0.07 \mathrm{ml} /$ injection, $10 \mathrm{mg} / \mathrm{ml}$ in physiological saline; Sigma, St. Louis, MO) in their hippocampus bilaterally (site 1, $1.0,0.7,2.0$; site $2,1.6,1.0,2.0$; site $3,1.6,2.0,2.5$; site $4,1.9,1.5$, 2.5 ; site 5, 1.9, 3.0, 3.5; site 6, 2.3, 2.0, 2.5; site 7, 2.3, 2.0, 3.0; site 8, 2.3, 3.5, 2.5; site 9, 2.3, 3.5, 3.0; site 10, 2.3, 3.5, 4.0; site $11,3.0,3.5,3.0$; site $12,3.5,3.0,3.0$; anteroposterior, lateral, and vertical distance, respectively, as measured from bregma, in millimeters). The sham-operated ( $\mathrm{SH}$ ) group received PBS $(\sim 0.06-0.07 \mathrm{ml} /$ injection $)$ in the same points. The surgery for eyeblink conditioning was made immediately after these lesion procedures. All mice recovered from surgery within $24 \mathrm{~h}$ and showed no signs of health problems or abnormal motor behavior. Behavioral tests took place after $7 \mathrm{~d}$ of recovery from surgery. After completing the behavioral tests, all mice were deeply anesthetized and perfused transcardially with $4 \%$ paraformaldehyde in PBS. Their brains were removed, postfixed overnight, and subsequently equilibrated in $30 \%$ sucrose solution. Coronal sections ( $40 \mu \mathrm{m}$ thick) were made to cover the entire hippocampus and stained with $1 \%$ cresyl violet. Images were digitally imported to image analysis system (Fluoview; Olympus, Tokyo, Japan). To estimate the extent of hippocampal damage in HIP subjects, we compared the length of cell layers of HIP subjects with that of the SH group (Cho et al., 1999). The mice in which $>5 \mathrm{~mm}$ of the cell layers was ablated were used for additional analysis.

\section{Eyeblink classical conditioning}

Surgery. Mice were anesthetized by ketamine $(80 \mathrm{mg} / \mathrm{kg}$, i.p.; Sankyo, Tokyo, Japan) and xylazine (20 mg/kg, i.p.; Bayer, Tokyo, Japan). Two stainless-steel screws were implanted into the skull, and a drop of dental cement was placed on the skull and around the screws. A strip connector with four Teflon-coated stainless-steel wires (75 $\mu \mathrm{m}$ in bare diameter; A-M Systems, Sequim, WA) was implanted on the skull and the wires subcutaneously implanted under the left eyelid. Two wires were used to record electromyogram (EMG) in the orbicuralis oculi muscle, which is responsible for eyelid closure. The remaining two were used to deliver the US. The connector was cemented to the skull screws with dental cement.

\section{Conditioning procedures}

A $352 \mathrm{~ms}$ tone $(1 \mathrm{kHz}, 80 \mathrm{~dB})$ was used as a CS, and $100 \mathrm{~ms}$ periorbital electrical shock (100 Hz square pulses) was used as US in all paradigms. In the delay conditioning, the US was given to overlap the CS in time such that the two stimuli terminate simultaneously. Delay conditioning experiment consisted of $7 \mathrm{~d}$ acquisition and $4 \mathrm{~d}$ extinction phases. In the trace conditioning, the US started $500 \mathrm{~ms}$ after the termination of the CS. Trace conditioning experiment consisted of $10 \mathrm{~d}$ acquisition and $4 \mathrm{~d}$ extinction phases. A daily training session consisted of 100 trials grouped in 10 blocks. The acquisition session consisted of 10 CS-only (every 10th trial) and 90 CS-US paired trials, whereas the extinction session consisted of 100 CS-only trials. Intertrial interval was randomized between 20 and $40 \mathrm{~s}$, with mean of $30 \mathrm{~s}$.

In all experiments, the US intensity was carefully determined as the minimal current amplitude required to elicit an eyeblink/head-turning response, which was adjusted daily for each animal. Experiments were performed during the light phase of the light/dark cycle in a container (10 $\mathrm{cm}$ in diameter) placed in a sound- and light-attenuating chamber. All of the procedures, including the surgery, were performed by the operator who was critically blind to the genotype of the mice.

\section{Data analysis}

The EMG was bandpass filtered between 0.15 and $1.0 \mathrm{kHz}$ and fed into a computer with a sampling rate of $10 \mathrm{kHz}$. The data for each session was processed off-line as follows. First, the maximum amplitude of the EMG signals during a time period of $t \pm 1 \mathrm{~ms}$ was calculated and denoted the EMG amplitude at $t$. Second, EMG amplitude values obtained from daily 100 trials for $300 \mathrm{~ms}$ before the CS onset were averaged, and the SD was calculated. Third, the average EMG value plus SD was defined as the threshold. Fourth, in each trial, EMG amplitude data for $300 \mathrm{~ms}$ before CS onset over the threshold were averaged and called the "Pre" value. The Startle value was calculated in the same way for $30 \mathrm{~ms}$ after the CS onset, and the trial was regarded as startle response if the startle value exceeded $30 \%$ of threshold. The CR value was usually calculated from the data for the last $200 \mathrm{~ms}$ before the US onset in the CS-US paired trials (adaptive CR). In the case of "early," CR value was calculated by a period when a CS tone is applied except for the first $52 \mathrm{~ms}$. The time window was extended 100 ms to obtain the CR value in the CS-only trials. Fifth, valid trials were defined as those with Pre and Startle values of $<10$ and $30 \%$ of the threshold, respectively. Sixth, a trial in which the CR value exceeded $1 \%$ of the threshold and exceeded at least twice the Pre value was regarded as a successful CR trial. Seventh, the ratio of successful CR trials to valid trials was calculated and denoted the CR (\%).

The response EMG was also analyzed by several parameters. The "averaged EMG" was analyzed by averaging EMG of individual mice over the valid trials and further averaged for each genotype. The averaged amplitude of response (see Fig. 5B) was quantitatively determined by averaging individual signal amplitudes of averaged EMG over the time points of early or adaptive phase. The frequency (see Figs. 2, 6) of the spontaneous eyeblink (sp) was measured during $100 \mathrm{CS}$ - and US-free trials of acclimation before the conditioning began. The startle response to a tone (supplemental Fig. $1 B$, available at www.jneurosci.org as supplemental material) was measured during the first session of trace conditioning. In pseudoconditioning (supplemental Fig. $1 E$, available at www.jneurosci.org as supplemental material), the CS and US were pseudorandomly presented with an interstimulus interval ranging from 0 to $20 \mathrm{~s}$.

\section{Other behavioral tests}

Rot-a-rod test. The rot-a-rod test (supplemental Fig. $1 A$, available at www.jneurosci.org as supplemental material) was performed as described previously (Kadotani et al., 1996). The rot-a-rod (Muromachi Kikai, Kyoto, Japan) consisted of a gritted metal roller $(25 \mathrm{rpm} ; 3 \mathrm{~cm}$ in diameter). A mouse was placed on the roller, and the time it remained on 
the rotating roller was measured. A maximum of $120 \mathrm{~s}$ was allowed for each animal.

Nociceptive tail-flick test. Nociception was measured according to the previous procedure (Bao et al., 1998). The latency to tail flicking on a hotplate (Ugo Basile, Comerio, Italy) at $51^{\circ} \mathrm{C}$ was measured three times at $1 \mathrm{~min}$ intervals.

\section{Statistical analysis}

Data were statistically analyzed by repeatedmeasures ANOVA following post hoc Scheffé's test with the SPSS 6.1 program (SPSS, Chicago, IL). Results shown are mean \pm SEM. The difference was considered significant when $p$ was $<0.05$.

\section{Results}

Intact hippocampus is required for trace but not delay eyeblink conditioning in wild-type mice

First, to investigate the hippocampal dependency of eyeblink conditioning in mice, the effect of bilateral hippocampal lesions by ibotenate was examined in C57BL/6 mice. As shown previously (Gerlai, 1998), injection of ibotenate resulted in severe hippocampal degeneration by axon swelling within both dorsal and ventral regions (Fig. 1A). In delay eyeblink conditioning, in which the CS and US coterminated, the percentage of conditioned eyeblink responses, which appeared in the last $200 \mathrm{~ms}$ period before the onset of US (referred to CR\%), was clearly increased for both $\mathrm{SH}$ and HIP groups (Fig. $1 B$ ) (group $\times$ session effect, $F_{(13,98)}=0.229, p=0.966$; group effect, $\left.F_{(1,14)}=0.148, p=0.706\right)$. We also measured averaged EMG amplitude of the eyelid response (Fig. 1C). No apparent difference was observed in the temporal property of CR between the two groups.

In contrast, in trace conditioning with a $500 \mathrm{~ms}$ stimulus-free interval (trace interval), hippocampal lesioned mice exhibited severely impaired acquisition of CRs during the $10 \mathrm{~d}$ training $($ Fig. $1 D)\left(\right.$ group $\times$ session effect, $F_{(19,140)}=2.0013, p=0.044$; group effect, $\left.F_{(1,14)}=87.232, p<10^{-6}\right)$. Averaged amplitude of eyelid responses on day 10 (Fig. $1 E$ ) indicated that the shamoperated mice exhibited both short-latency responses $\sim 50 \mathrm{~ms}$ after the CS onset and long-latency adaptive CRs, which were elicited after the termination of CS, as described previously (Kishimoto et al., 2001, 2002). In the hippocampus-lesioned mice, this short-latency response remained to some degree, but almost no adaptive CRs were observed. We confirmed the sensitivity to the US, and the minimum US intensity required to elicit the eyelid response of the hippocampus-lesioned mice was nearly the same as that required for sham-operated mice. The eyelid response during the pseudoconditioning, in which the order of CS and US were randomized, did not increase in either group (data not shown). We thus confirmed that the $500 \mathrm{~ms}$ interval trace eyeblink conditioning is hippocampus dependent in the wild-type mice, whereas the delay conditioning is hippocampus independent.

Normal acquisition but impaired extinction of trace eyeblink conditioning in CA3-NR1 KO mice

We next subjected CA3-NR1 KO mice and their control floxedNR1 littermates to the same trace conditioning paradigm as de-
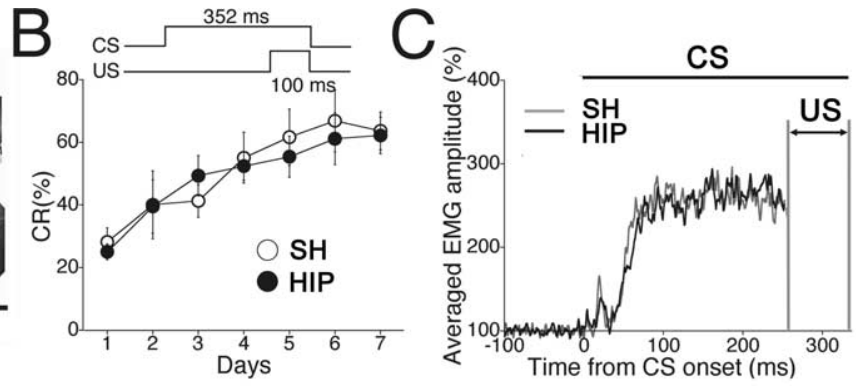

$E$

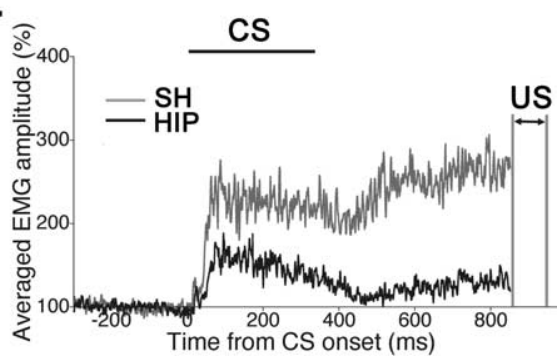

US

Figure 1. Effect of bilateral hippocampal lesion on delay and trace eyeblink conditioning in $C 57 \mathrm{BL} / 6 \mathrm{mice}$. $\boldsymbol{A}$, Representative coronal sections showing the hippocampal formation in ibotenate-injected wild-type mice. Nissl-stained coronal sections of yeblink conditioning in both SH and HIP mice B Percentages of conditioned response (CR\%) were increased in both SH (open (black trace) mice on day 7, indicating Temporal relationship between the CS and US is shown in the top panel. $\boldsymbol{E}$, Average EMG amplitude on day 10 of the SH mice (gray trace) showed adaptive CRs in a biphasic conditioned response. In contrast, HIP mice (black trace) showed almost no adaptive CRs, whereas diminished short-latency CRs still remained.

scribed above. The CR (\%) for both groups progressively increased up to $60 \%$ during the acquisition phase, and no difference was observed between the control and mutant mice (Fig. 2A) (group $\times$ session effect, $F_{(19,210)}=0.383, p=0.942$; genotypic effect, $\left.F_{(1,21)}=0.466, p=0.502\right)$. In contrast, mutant mice exhibited higher $\mathrm{CR}(\%)$ than control mice on day 11 in the extinction phase (Fig. $2 A$ ) (group $\times$ session effect, $F_{(7,84)}=$ 2.543, $p=0.064$; genotype effect, $\left.F_{(1,21)}=12.230, p=0.0021\right)$. However, this deficit almost disappeared on day 12, suggesting the impairment is in the initial rate of extinction learning. EMG topographies of individual mice for 10-trial blocks on day 10 indicate that there was no difference in the response amplitudes between the two genotypes (Fig. $2 \mathrm{~B}$ ). In contrast, highermagnitude responses were observed in CA3-NR1 KO mice compared with control mice on day 11 (Fig. 2B). The augmented EMG amplitudes in day 11 were prominent in the early phase of CRs within each trial (Fig. 3A, Day 11), especially during the second and subsequent 10-trial blocks (Fig. 5A, Day 11). These results suggest that the acquisition of trace memory is normal, but the extinction is retarded in CA3-NR1 KO mice.

\section{Mutant conditioned response amplitudes were augmented during extinction of trace conditioning}

The CR in trace eyeblink conditioning includes eyelid closure that is precisely timed to occur just before the time point when the US would have been delivered during the training session (Gormezano et al., 1987). To characterize the temporal features of eyeblink response, the amplitudes of response EMGs were averaged for each day throughout the acquisition and extinction phases (Fig. 3A). The amplitudes of short-latency responses in the early phase of CRs increased from day 1 to day 5 . These 

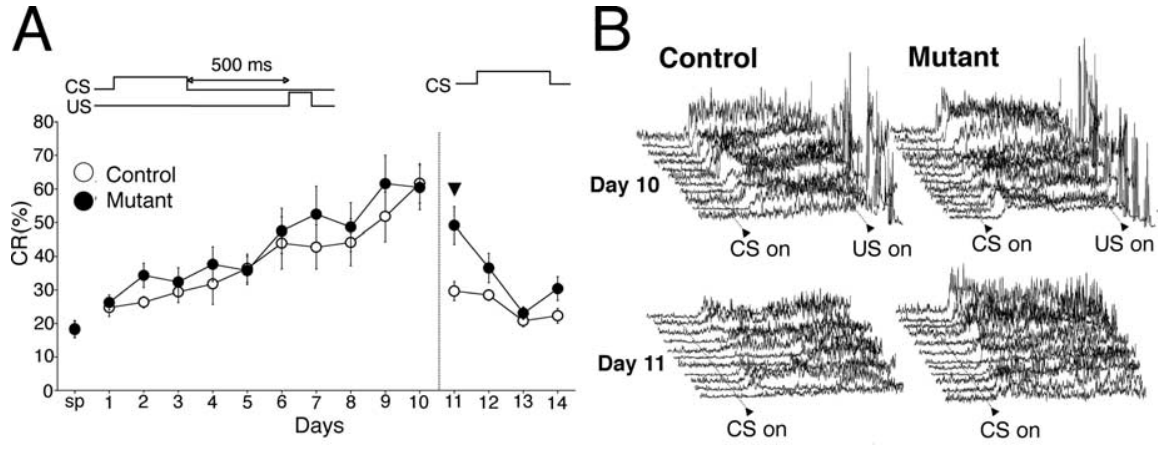

Figure 2. Normal acquisition and impaired extinction of adaptive CRs during trace conditioning in CA3-NR1 K0 mice. A, Trace conditioning experiment consisted of an acquisition phase (days 1-10) followed by an extinction phase (days 11-14). In the acquisition phase, there was no difference in the percentage of adaptive CRs evaluated daily, which were elicited within $200 \mathrm{~ms}$ of the US onset during trace conditioning between floxed-NR1 (control; open circles; $n=11$ ) and CA3-NR1 K0 (mutant; closed circles; $n=12$ ) mice. In contrast, the mutant CR (\%) was significantly higher than that of controls in the extinction phase. Temporal relationships between CS and US are imposed in the top panel. sp, Spontaneous eyeblink response. The arrowhead indicates significant difference between the control and mutant mice. $\boldsymbol{B}$, EMG response topographies of individual mice averaged by each 10-trial block on day 10 (last session of acquisition phase) and day 11 (first session of extinction phase). On day 10, no difference was observed between the control (left) and mutant (right) mice. However, on day 11 , the response amplitudes of the mutant mice were significantly larger than those of the controls (Fig. 3B, day 11).
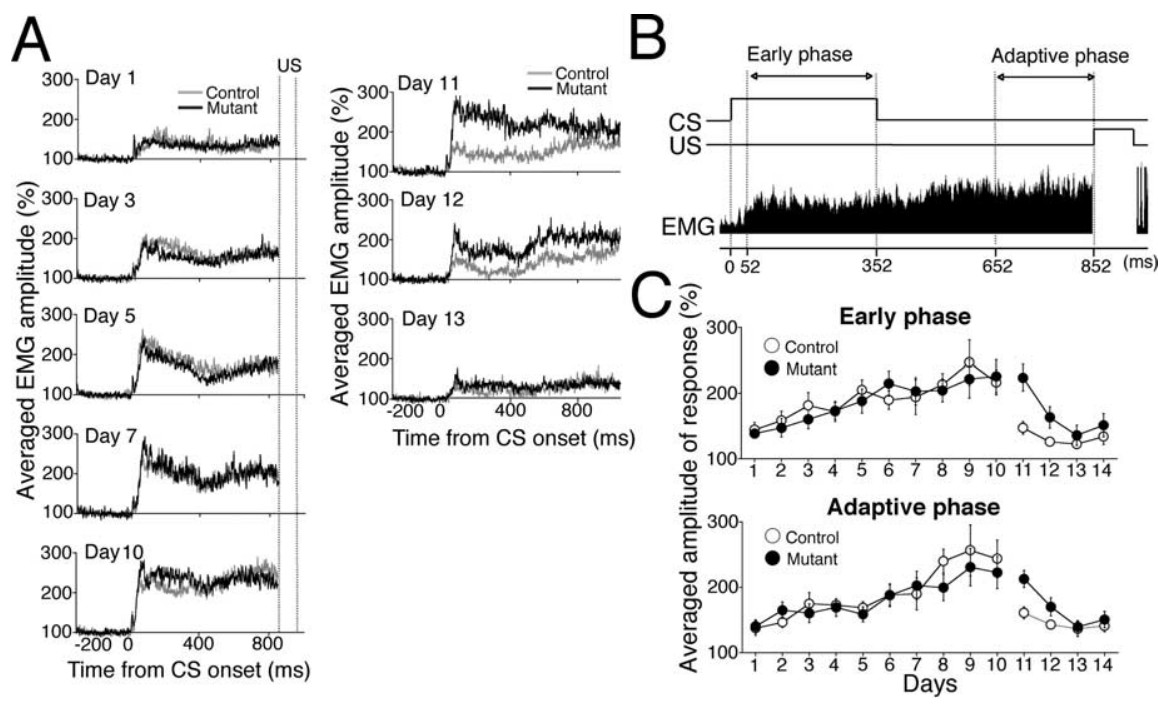

Figure 3. Impaired adaptive CR during extinction of trace conditioning in CA3-NR1 KO mice. A, Left, Response EMGs for both the control (gray traces) and the mutant (black traces) mice were averaged daily on days 1,3,5,7, and 10 during acquisition phase in trace conditioning, and there were no differences in any of the days between the two genotypes. Right, During extinction phase, significantly higher response amplitudes of average EMG were observed in mutants than in controls on days 11 and 12 but not on day 13. $\boldsymbol{B}$, An example of response EMG from control mice in a CS-US paired trial is presented. Averaged amplitudes of EMG were evaluated by the two distinct phases. Early phase was defined as a period when a $C S$ tone is applied except for the first $52 \mathrm{~ms}$. Adaptive phase was a period of 200 ms before the US. C, Averaged amplitudes of response EMGs during the early (top) and adaptive (bottom) phases are calculated throughout the entire acquisition and extinction sessions. The mutants showed higher levels of EMG amplitudes in both early and adaptive phases on day 11 , suggesting extinction of both adaptive and nonadaptive CR was retarded in CA3-NR1 K0 mice.

amplitudes seemed to reach the maximal level at approximately day 5 in both control and mutant mice (Fig. $3 A$ ). In contrast, the amplitudes of the long-latency response continued to increase after day 5 in both genotypes. To quantify these EMG amplitude changes, we set up two periods during the conditioned eyelid response: early phase, spanning $300 \mathrm{~ms}$ from 52 to $352 \mathrm{~ms}$ after the CS onset; and adaptive phase, spanning $200 \mathrm{~ms}$ from 652 to $852 \mathrm{~ms}$ (Fig. 3B). There was no significant difference in the dayto-day patterns of averaged EMG amplitudes between the mutant and control mice throughout acquisition for both phases (group $\times$ session effect, $F_{(19,210)}=0.405, p=0.932$; genotypic effect, $F_{(1,21)}=0.087, p=0.577$ for early phase; group $\times$ session effect, $F_{(19,210)}=$ $0.564, p=0.825$; genotypic effect, $F_{(1,21)}=$ $0.309, p=0.584$ for adaptive phase) (Fig. $3 C)$. In contrast, on days 11 and 12 in the extinction phase, the EMG amplitudes were greater in mutant mice for both phases compared with the controls (group $\times$ session effect, $F_{(7,84)}=2.767$, $p=0.049$; genotype effect, $F_{(1,21)}=7.345$, $p=0.013$ for early phase; group $\times$ session effect, $F_{(7,84)}=1.836, p=0.149$; genotype effect, $F_{(1,21)}=10.048, p=0.0046$ for adaptive phase) (Fig. 3C).

Together, the data in Figures 2 and 3 suggest the impaired initiation of adaptive CR extinction. Moreover, because extinction of the CR in the early phase, as measured by EMG amplitude, was also impaired in the CA3-NR1 KO mice, we concluded that extinction of both adaptive and nonadaptive CRs were retarded in CA3-NR1 KO mice.

\section{Impaired adaptively timed CR} frequency in the first 10 trials of daily acquisition in CA3-NR1 KO mice To examine in more detail whether the mutants are normal in memory acquisition or storage of the adaptive CR, we calculated the frequency of the adaptive CR (\%) in 10-trial blocks during the daily acquisition sessions (Fig. 4A). Although the CR (\%) of the control mice increased to $\sim 70 \%$ in the first 10 trials (1st to 10th trials) of acquisition phase, CR (\%) of mutants reached $40 \%$ on day 4 and ceased to increase further (group $\times$ session effect, $F_{(19,210)}=2.613, p=0.007$; genotypic effect, $F_{(1,21)}^{(19,210)}=1.648, p=0.213$ ) (Fig. $4 B$, right). On days $8-10$, the mutants' CR (\%) was significantly smaller in the first 10 trials than the control CR (\%). This impairment was not seen during the second 10 trials (11-20th trials) (group $\times$ session effect, $F_{(19,210)}=0.226, p=0.991$; genotypic effect, $\left.F_{(1,21)}=0.924, p=0.348\right)$ nor during any of the subsequent 10 trial segments, as exemplified by the final 10 trial segment (group $\times$ session effect, $F_{(19,210)}=0.715$, $p=0.695$; genotypic effect, $F_{(1,21)}=1.569$, $p=0.224)$. In contrast, CA3-NR1 KO mice showed normal acquisition of CR (\%) during the early phase even within trials $1-10$ (group $\times$ session effect, $F_{(19,210)}=$ $0.325, p=0.966$; genotypic effect, $F_{(1,21)}=0.274, p=0.606$ ) (Fig. $4 B$, left). These results suggest that CA3-NR1 KO mice are able to acquire the memory of the CS-US association, but they could not demonstrate it as adaptively timed CRs in the first 10 trials of acquisition phase.

To further evaluate the impaired deficit in adaptive CR (\%) during the first 10 trials, we directly compared adaptive CRs (\%) of the last 10 trials and the first 10 trials of the next day during the last $3 \mathrm{~d}$ of the acquisition phase (Fig. 4C). Control mice did not 
show any reduction in the first 10 -trial CR (\%) on the next day during these $3 \mathrm{~d}$, indicating that memory acquired by the previous day in the control mice was successfully retrieved from the beginning of the following day. In contrast, mutant mice showed significant reductions of CR (\%) in the first 10 trials compared with that of the last 10 trials of the previous day on days 9 and $10(p=0.037$ and 0.046 , respectively), confirming the reduced adaptive CR memory in the first 10 trials of the mutant animals.

To determine how many trials were required for the mutants' memory to catch up to the level of adaptive CRs relative to controls, we further calculated averaged CR (\%) with every one-trial within the first 20 trials from days 8 to 10 (Fig. $4 D$ ). We found that the CR (\%) of the controls was $>50 \%$ in the first trial, and no significant increase was observed in the following trials, indicating that the performance of trace conditioning in the controls already reached the asymptotic level in the first trial. In contrast, the averaged CR (\%) of the mutants was $<30 \%$ in the first three trials, which corresponded to the CR (\%) level of day 1 during acquisition (Fig. $2 A$ ). There is a statistically significant difference in CR (\%) between the two groups during the first five trials (group $\times$ session effect, $F_{(9.335)}=0.589, p=0.671$; genotypic effect, $\left.F_{(1,67)}^{(0,335)}=3.984, p=0.0003\right)$. These results suggest that the memory of adaptively timed CRs stored by the previous day is retrieved from the first trial in the controls after their learning reached the asymptotic level by day 7. In contrast, the mutants showed impairment in retrieving memory in the early trial sessions. Interestingly, this impaired memory in the mutant was restored from the sixth trial, indicating that adaptive $\mathrm{CR}$ memory is able to be stored without CA3 NRs by the repetitive CS-US association.

Is the reduced adaptive $\mathrm{CR}$ of the mutants during the first five trials of a day caused by a failure in the full transfer of the CR memory acquired by the previous day, or is it because of a deficit in the retrieval of the CR memory from the first trial, namely, rapid retrieval? Given that the CR memory could be recalled without US on day 11 , the animals' capability of transferring memory across days should be intact. We evaluated adaptive CR (\%) in the first day of extinction phase (day 11) with every one-trial (Fig. 4E). Control CR (\%) was decreased throughout the 100-trials session and reached the level of day 1 in the last 20 trials (91-100th trials). In contrast, the mutants maintained the CR (\%) level of the early trials until the final trial of the session. In the last 10 trials, the mutant CR level

C

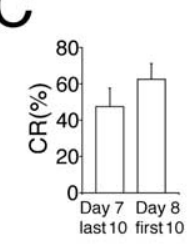

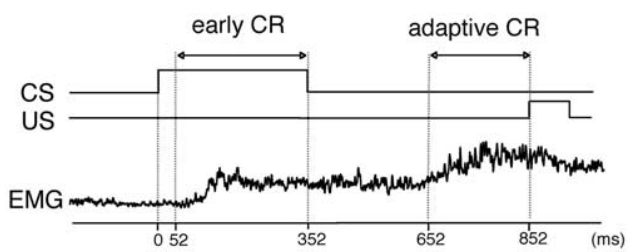
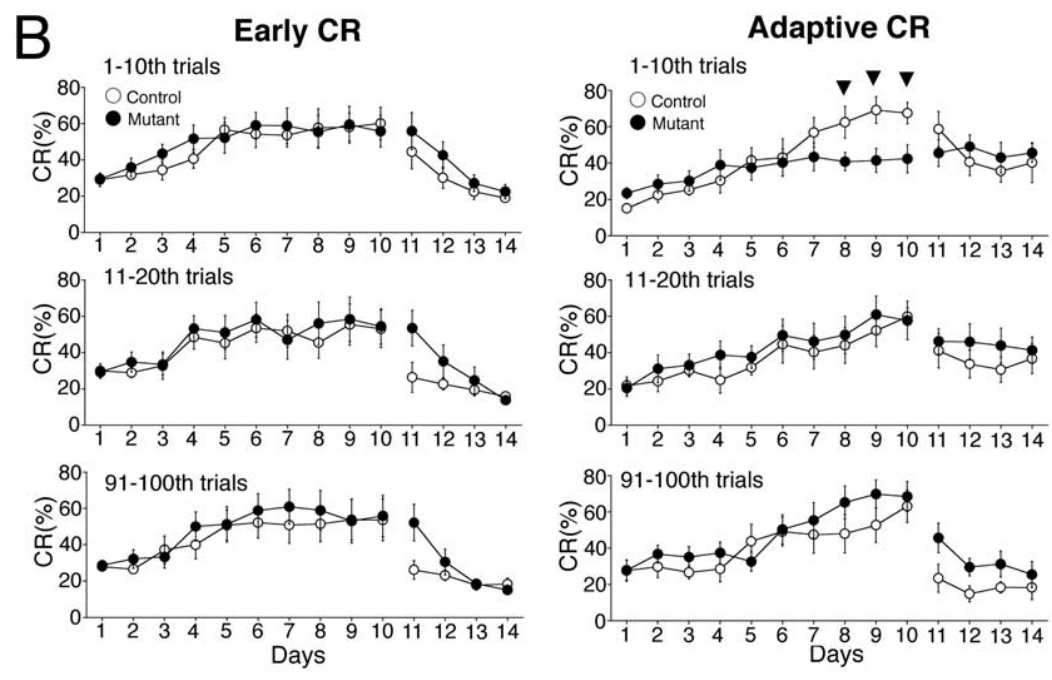

Control
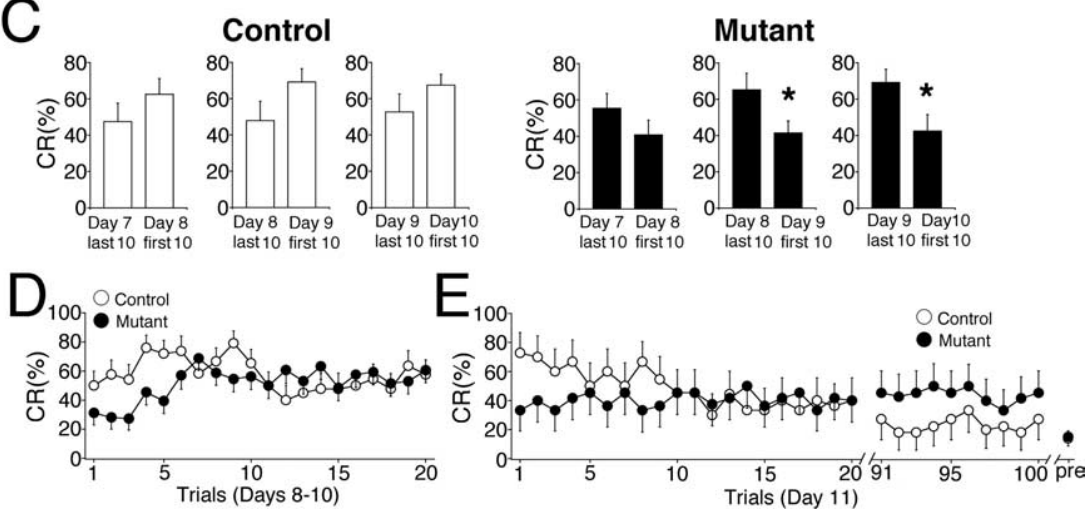

Figure 4. Frequency of adaptive CR was impaired within the first 10 trials of acquisition in CA3-NR1 K0 mice. $A$, An example of typical CR from control mice in a CS-US paired trial is presented. Frequency of CR (\%) was evaluated by the two distinct time windows. Adaptive CR was judged by the response EMG data for the last $200 \mathrm{~ms}$ before the US. Early CR was evaluated by a period when a CS tone is applied except for the first $52 \mathrm{~ms}$. B, Left, Early CRs (\% frequency) of both control (open circles) and mutant (closed circles) mice were evaluated in the first 10 trials (1-10th trials; top), the second 10 trials (11-20th trials; middle), and the final 10 trials (91-100th trials; bottom) of daily session during trace conditioning. There was no difference in any blocks between two genotypes, whereas the mutant CR (\%) was higher in the last 10-trial block on day 11. Right, Adaptive CR (\% frequency) was averaged every 10 -trial block in both groups. The mutant $C R(\%)$ in the first 10 trials from days 8 to 10 was significantly higher than that of controls. The higher mutant CR (\%) was also observed in the last 10 trials on day 11 during extinction. C, Comparison of adaptive CRs (\%) between the last 10 trials (91-100th trials) of a session and the first 10 trials (trials 1-10) of the session on the following day during the last 4 days of acquisition phase. There was no significant change of adaptive $C R(\%)$ in the control mice from the last 10 trials on days 7,8 , and 9 to the first 10 trials on days 8,9 , and 10 , respectively. In contrast, the mutants exhibited significant reduction in adaptive $C R(\%)$ from the last 10 trials on days 8 and 9 to the first 10 trials on days 9 and 10 , respectively. $D$, Adaptive CRs (\% frequency) of two genotype animals on days 8,9, and 10 during trace conditioning were averaged on each trial (trials 1-20). In the first three trials, the level of mutant (Rs was nearly the same as that observed on day 1. However, it was restored until the sixth trial, and no difference was observed hereafter between two groups. $\boldsymbol{E}$, Adaptive (Rs (\%) on the first day of extinction (day 11) were averaged on every trial (trials 1-20 and 91-100) for each genotype. Control mice exhibited a gradual decrease in $\mathrm{CR}(\%)$ throughout the 100-trials session, demonstrating the normal extinction curve. In contrast, $\mathrm{CR}(\%)$ in the mutant mice did not change within the session. As a result, a significant amount of adaptive CR memory remained in the last 10 trials. pre, Averaged frequency of eyeblink response before the training for acquisition (background).

was significantly higher than the background CR level (Fig. $4 E$, pre), which was measured before the training for acquisition (group $\times$ session effect, $F_{(39,420)}=0.093, p=0.999$; genotypic effect, $\left.F_{(1,21)}=4.775, p=0.041\right)$. These CRs (\%) of the mutants 
A
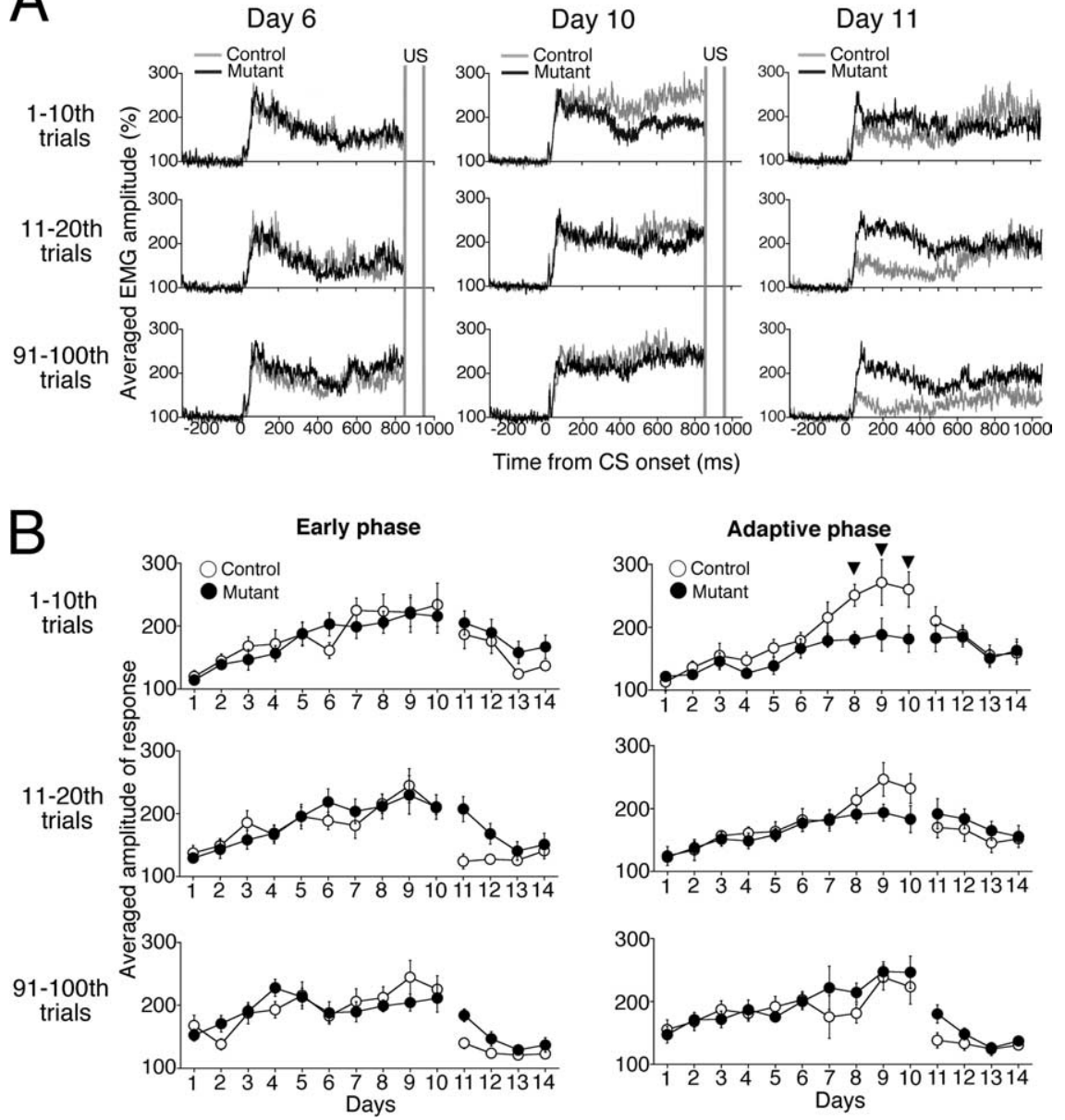
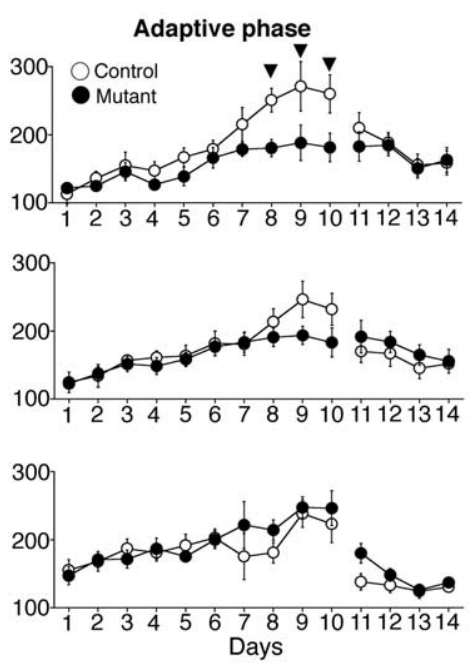

Figure 5. Impaired elevation of adaptive EMG amplitude in the first 10 trials of acquisition in CA3-NR1 K0 mice. $A$, Response EMG (amplitude \%) of both control (gray traces) and mutant (black traces) mice is shown with the first 10 trials (1-10th trials; top), the second 10 trials (11-20th trials; middle), and the final 10 trials (91-100th trials; bottom) of the daily session on days 6 and 10 (acquisition phase) and on day 11 (extinction phase) of trace conditioning. The adaptive (R amplitude in the first 10 trials on day 10 of the mutants was significantly lower than that of controls. The higher short-latency CR amplitude was also observed in trials 11-20 and the last 10 trials on day 11 in the mutant mice. $\boldsymbol{B}$, Averaged amplitudes of response EMGs during the early (left) and adaptive (right) phases are presented with the first 10 trials (top), the 11-20th trials (middle), and the 91-100th trials (bottom) of daily sessions, respectively. Mutants failed to show an increase in the adaptive phase-EMG amplitudes to the level of controls in the first 10 trials from days 8 to 10 . Arrowheads indicate significant difference in $C R(\%)$ between the control and mutant mice.

were also greater than the CRs $(\%)$ of the controls (group $\times$ session effect, $F_{(39,420)}=-1.95$; genotypic effect, $F_{(1,21)}=5.059$, $p=0.035)$. These results showed the remnants of CR memory in the mutants even after extinction trials on day 11 . Therefore, the mutants' impaired CR (\%) during the first five trials in the acquisition phase should not be attributable to the deficit in the carryover of memory from the previous day. It is noted that the impaired extinction resulted in the demonstration of successful memory transfer from day 10 to day 11 in the mutant animals.

\section{Impaired adaptively timed CR in the first 10 trials of daily acquisition in CA3-NR1 KO mice}

To examine whether the temporal property of the adaptive EMG response is also defective in the mutants, averaged amplitudes in 10 -trial blocks were plotted throughout the acquisition and extinction phases. Up to day 6 , there was no temporal difference of EMG amplitudes between the two genotypes (Fig. 5A). During the trials on day 6, the EMG amplitudes during the adaptive phase increased gradually in both mutants and controls (see the last 10 trials on day 6). On day 10, the amplitudes of the adaptive CRs of the control mice were clearly greater than those on earlier days such as day 6 in all 10-trial blocks. In contrast, the mutant mice exhibited lower amplitudes of adaptive CRs in the first 10 trials. The EMG amplitudes for the mutants' adaptive CRs became larger by the second 10 -trial block on day 10 , and the same amplitude level as that of controls was maintained until the last 10-trial block.

In the extinction phase, control EMG amplitudes decreased from the first 10 trials to the last 10 trials on day 11, whereas the mutant CR amplitudes did not decrease (Fig. 4A). Interestingly, the averaged EMG amplitudes in the last $500 \mathrm{~ms}$ before US onset were still high in the mutants, suggesting that the adaptive CR memory was preserved even after the extinction phase began.

We quantified the impairment in the EMG amplitudes by evaluating each 10trial block daily (Fig. 5B). In the first 10 trials (1-10th trials) of acquisition sessions, the mutants' lower amplitude responses relative to the control mice were observed only in the adaptive CR phase (on days 8-10) (group $\times$ session effect, $F_{(19,210)}=2.004, p=0.041$; genotypic effect, $\left.F_{(1,21)}=7.127, p=0.014\right)$. This impairment was restored from the second 10 trials (group $\times$ session effect, $F_{(19,210)}=$ $0.562, p=0.827$; genotypic effect, $F_{(1,21)}=$ $1.754, p=0.199)$. There was no difference in any of the 10-trial blocks during the early phase between the two groups, again indicating that the memory impairment is specific to the timing of the adaptive CR during the first 10 trials. These results suggest that the mutants are unable to retrieve the adaptive CR memory in the early stage of daily sessions. Nevertheless, in the following trials, they can rapidly restore the response timing learned by the preceding sessions of CS-US paired trials.

In trials 91-100 of extinction phase, the averaged response amplitude was higher in the mutant mice than in the control mice (Fig. 5B). In trials 11-20, the mutant's higher amplitude responses in the early phase was more pronounced (group $\times$ session effect, $F_{(7,84)}=2.468, p=0.071$; genotype effect, $F_{(1,21)}=$ $7.238, p=0.0014$; in trials $11-20$ of the early phase). Together with the data in Figure $4 B$, these results suggest that the initiation of extinction was retarded in the mutants, in particular for the memory of the early phase of CRs.

\section{Normal delay eyeblink conditioning in CA3-NR1 KO mice}

We next examined the performance of CA3-NR1 KO mice on delay eyeblink conditioning by using different mice from those subjected to trace eyeblink conditioning. The CR (\%) for both CA3-NR1 KO mice and their control littermates progressively increased up to $\sim 70 \%$ during the $7 \mathrm{~d}$ acquisition training (Fig. $6 A)$. During the following $4 \mathrm{~d}$ extinction phase, CR (\%) de- 
creased similarly in control and mutant mice (Fig. 6A). Individual EMG topographies on day 7 (Fig. $6 A$, top inset) demonstrate that there was no difference in the response amplitudes between the two genotypes. These results suggest that acquisition and extinction of CRs for delay conditioning is normal in CA3-NR1 KO mice (group $\times$ session effect, $F_{(13,147)}=2.160$, $p=0.051$; genotypic effect, $F_{(1,21)}=0.083$, $p=0.777$ for acquisition phase; group $\times$ session effect, $F_{(7,84)}=0.555, p=0.647$; genotypic effect, $F_{(1,21)}=0.221, p=0.643$ for extinction phase). The peak CR latencies for both genotypes were constant throughout acquisition and extinction phases, and there was no significant difference between genotypes in this measure (group $\times$ session effect, $F_{(13,147)}=0.012$, $p=0.999$; genotypic effect, $F_{(1,21)}=0.052$, $p=0.822$ for acquisition phase; group $\times$ session effect, $F_{(7,84)}=0.176, p=0.913$; genotypic effect, $F_{(1,21)}=0.0009, p=$ 0.976 for extinction phase). The averaged amplitudes and temporal properties of CRs of the mutant mice on day 7 were nearly the same as those of the control mice (Fig. 6B). CA3-NR1 KO mice exhibited normal CR (\%) within the first 10 trials of each daily session (group $\times$ session effect, $F_{(13,147)}=0.624, p=0.710$; genotypic effect, $\left.F_{(1,21)}=0.011, p=0.919\right)$ as well as within the 11-20th trials (group $X$ session effect, $F_{(13,147)}=0.442, p=0.850$; genotypic effect, $F_{(1,21)}=1.096, p=$ $0.307)$ and the 91-100th trials (group $\times$ session effect, $F_{(13,147)}=$ $1.180, p=0.321$; genotypic effect, $\left.F_{(1,21)}=0.633, p=0.435\right)$ during the acquisition phase (Fig. $6 C$ ). These results suggest that adaptively timed CRs during delay conditioning were successfully formed in both intra-day and across-day time scales in CA3NR1 KO mice. These results suggest that the mechanisms for exhibiting CRs for the delay conditioning are intact in CA3-NR1 $\mathrm{KO}$ mice.

\section{Eyeblink sensory motor function is normal in CA3-NR1 KO mice}

We further investigated the motor and sensory function of CA3NR1 KO mice. As detailed in supplemental Figure 1 (available at www.jneurosci.org as supplemental material), mutant mice exhibited normal motor coordination, startle response, and tail flick response. There was no difference between control and mutant mice in the minimum stimulus intensity used for the US during trace conditioning. Moreover, CR (\%) did not improve during the pseudoconditioning procedure in either genotype. These results show that CA3-NR1 KO mice are not impaired in motor coordination and sensory functions required to learn eyeblink conditioning.

\section{Discussion}

It has been shown previously that eyeblink conditioning with a long stimulus-free interval of 250-1000 ms (trace conditioning) is sensitive to hippocampal lesion (Solomon et al., 1986; Moyer et al., 1990; Kim et al., 1995; McGlinchey-Berroth et al., 1997; Clark and Squire, 1998; Weiss et al., 1999; Tseng et al., 2004; Sakamoto et al., 2005), whereas the acquisition of conditioning with no stimulus-free interval (delay conditioning) can occur without the intact hippocampus. In the present study, we confirmed distinction in hippocampal-lesioned C57BL/6 mice given either delay or trace (500 ms trace interval) conditioning. We then analyzed the CA3-NR1 KO mice in which the NR1 gene knock-out was mostly restricted to the CA3 pyramidal cells of adult mice.

We found that these mutant mice can acquire both nonadaptive (early) and adaptive (late) CRs during hippocampusdependent trace eyeblink conditioning when they were assessed by both their frequencies and EMG amplitudes averaged over a block of 100 daily trials. However, as the training advanced (starting on day 8), the adaptive CRs were significantly reduced in mutants compared with control mice during the first several trials of the day. In addition, mutants exhibited impairments in extinction of both adaptive and nonadaptive CRs.

\section{A specific role of CA3 NMDA receptors in adaptive CR}

Our analysis of CA3-NR1 KO and control mice revealed at least two distinct types of CR memory that can be formed and recalled in control mice during trace eyeblink conditioning. CRs based on the first type of memory (early CR) start to appear rapidly after the onset of CS and recede slowly during and after CS. This type of CR memory is apparently independent of CA3 NR function. The appearance of CRs based on the second type of memory (adaptive CR) is delayed until the onset of the trace period and seems to be composed of both CA3-NR-dependent and -independent components. 
Rodriguez and Levy (2001) theorized that the CA3 network could play a crucial role in the acquisition of adaptive CRs by serial activation of CA3 neurons during the trace period through the recurrent dynamics of these cells and temporally asymmetric synaptic modification mechanisms (Rodriguez and Levy, 2001). Our results are consistent with this theory as far as the CA3-NRdependent adaptive $\mathrm{CR}$ is concerned. However, the finding of the CA3-NR-independent component of the adaptive CR, the third type of memory, cannot be accounted for by Rodriguez and Levy's (2001) theory and suggests the presence of an alternative storage site of adaptive CRs in the hippocampus.

\section{Model of adaptive CR memory}

Using the same CA3-NR1 KO mice, we showed previously that CA3 NRs, unlike CA1 NRs, are dispensable for the acquisition of spatial reference memory or for its recall under full cue conditions but are crucial for the recall of the same memory under partial cue conditions (i.e., recall by pattern completion) (Nakazawa et al., 2002). We assumed that the memory traces were formed both in the CA1 and CA3 networks in the control mice. In CA3-NR1 KO mice, the memory trace would be formed in the CA1 network but not in the CA3 network. Consequently, the partial cue input cannot evoke the full-cue activity in CA3 by pattern completion, which is essential for full activation of the CA1 memory trace. However, the CA1 memory trace would be activated fully if the entire set of recall cues was provided.

We extend this model for a hippocampus-dependent spatial reference memory and its retrieval to the equally hippocampusdependent adaptive CR memory in trace eyeblink conditioning. The adaptive CR memory is acquired incrementally through repeated conditioning trials as the spatial reference memory is acquired through repeated training trials. We suggest that the NMDAR-dependent memory trace is formed in the CA3 recurrent network along with other hippocampal networks such as the CA1 network, and that once established, the CR memory can be recalled with a single CS as long as the CA3 recurrent network is intact. This is because, although the single CS per se may not be effective enough to fully reactivate the adaptive CR memory traces across the stimulus-free time gap (trace period), the iterative activity within the CA3 memory network will bridge this gap (Levy, 1996; Wallenstein et al., 1998) and complete the reactivation, permitting recall of CA3-NR-dependent adaptive CR memory. In the CA3-NR1 KO mice, this iterative activity in the CA3 network would be missing and, therefore, the mutants will have to rely on multiple rounds of CS to fully activate the CR memory trace stored outside of the CA3 network, such as the CA1 network, that is, recall of CA3-NR independent adaptive CR memory. According to this interpretation of our data, the CA3-NR in the recurrent network plays a crucial role not only in spatial pattern completion (Nakazawa et al., 2002) but also in temporal pattern completion.

\section{Impaired extinction of adaptive CR memory}

As much as extinction is a new learning (Pavlov, 1927; Myers and Davis, 2002), hippocampal NRs are involved in both acquisition and extinction of hippocampus-dependent memory. For instance, infusion of AP-5 into the hippocampus has been shown to block both acquisition and extinction of one-trial passive avoidance task (Izquierdo et al., 1999; Szapiro et al., 2003). A previous pharmacological study on rabbit eyeblink conditioning has also demonstrated that systemic infusion of (+)-5-methyl-10,11dihydro-5H-dibenzo [a,d] cyclohepten-5,10-imine maleate or phencyclidine blocks both acquisition and extinction of adaptive
CRs (Thompson and Disterhoft, 1997). Our present study showed that CA3-NR1 KO mice were significantly impaired in the first day (day 11) of adaptive CR extinction. However, this impairment was mostly alleviated on the following day (day 12). This is in line with our previous finding that the same mutant mice were impaired in a novelty-associated spatial task (Nakazawa et al., 2003) but not in the acquisition of spatial reference memory (Nakazawa et al., 2002).

Interestingly, CA3-NR1 KO mice were not impaired in the extinction of delay conditioning (Fig. 6A,C). Because systemic administration of NR antagonists has been shown previously to impair the extinction of the adaptive CRs in rats (Thompson and Disterhoft, 1997), the combined results suggest that extinction of delay conditioning is mediated by NR function outside of area CA3.

\section{CA3-NR1 KO mice are impaired in "internal inhibition"}

We observed a higher magnitude of individual EMG responses in the mutants after the onset of CS on day 11 (Fig. 2 B). In particular, the mutants showed a tendency to blink more robustly as a short-latency CR in response to the CS than control mice (Figs. $3 A, 5 A, B)$. This could be related to the previous observation showing that hippocampal animals are impaired in internal inhibition (Pavlov, 1927) or "behavioral inhibition" (Douglas, 1967; Kimble, 1968), which resulted in perseveration of the CR in response to the CS (Niki, 1966; Kim et al., 1995; Chan et al., 2001). These results suggest that CA3 NRs may function to exclude CS from attention to protect memory from interference. In fact, our preliminary data showed that CA3-NR1 KO mice are impaired in latent inhibition of eyeblink conditioning (Y. Kishimoto, unpublished data).

\section{Conclusions}

Our results demonstrated that CA3 NRs play a critical role in trace eyeblink conditioning, a form of nonspatial learning. Consistent with our previous result showing that acquisition of spatial reference memory is normal in the CA3-NR1 KO mice (Nakazawa et al., 2002), we did not observe obvious impairment in acquisition of trace conditioning. Our analyses of CR timing revealed, however, that CA3 NRs are required for the memory of adaptive CR timing, which is ready to be retrieved at any moment. The pivotal role of CA3 NRs in rapid retrieval of CR timing seems to be of particular importance for animals to respond adaptively to a daily episode in the ever-changing environment.

\section{References}

Abbott LF, Blum KI (1996) Functional significance of long-term potentiation for sequence learning and prediction. Cereb Cortex 6:406-416.

August DA, Levy WB (1999) Temporal sequence compression by an integrate-and-fire model of hippocampal area CA3. J Comput Neurosci 6:71-90.

Bao S, Chen L, Qiao X, Knusel B, Thompson RF (1998) Impaired eye-blink conditioning in waggler, a mutant mouse with cerebellar BDNF deficiency. Learn Mem 5:355-364.

Chan KH, Morell JR, Jarrard LE, Davidson TL (2001) Reconsideration of the role of the hippocampus in learned inhibition. Behav Brain Res 119:111-130.

Cho YH, Friedman E, Silva AJ (1999) Ibotanate lesions of the hippocampus impair spatial learning but not contextual fear conditioning in mice. Behav Brain Res 98:77-87.

Clark RE, Squire LR (1998) Classical conditioning and brain systems: the role of awareness. Science 280:77-81.

Douglas RJ (1967) The hippocampus and behavior. Psychol Bull $67: 416-422$.

Gerlai R (1998) Contextual learning and cue association in fear condition- 
ing in mice: a strain comparison and a lesion study. Behav Brain Res 95:191-203.

Gormezano I, Prokasy WF, Thompson RF (1987) Classical conditioning, Ed 3. Boca Raton, FL: CRC.

Hasselmo ME, McClelland JL (1999) Neural models of memory. Curr Opin Neurobiol 9:184-188.

Hasselmo ME, Schnell E, Barkai E (1995) Dynamics of learning and recall at excitatory recurrent synapses and cholinergic modulation in rat hippocampal region CA3. J Neurosci 15:5249-5262.

Hasselmo ME, Bodelon C, Wyble BP (2002) A proposed function for hippocampal theta rhythm: separate phases of encoding and retrieval enhance reversal of prior learning. Neural Comput 14:793-817.

Izquierdo I, Medina JH, Vianna MRM, Izquierdo LA, Barros DM (1999) Separate mechanism for short- and long-term memory. Behav Brain Res 103:1-11.

James GO, Hardiman MJ, Yeo CH (1987) Hippocampal lesions and trace conditioning in the rabbit. Behav Brain Res 23:109-116.

Jensen O, Lisman JE (1996) Hippocampal CA3 region predicts memory sequences: accounting for the phase precession of place cells. Learn Mem 3:279-287.

Kadotani H, Hirano T, Masugi M, Nakamura K, Nakao K, Katsuki M, Nakanishi S (1996) Motor discoordination results from combined gene disruption of the NMDA receptor NR2A and NR2C subunits, but not from single disruption of the NR2A or NR2C subunit. J Neurosci 16:7859-7867.

Kesner RP, Gilbert PE, Wallenstein GV (2000) Testing neural network models of memory with behavioral experiments. Curr Opin Neurobiol 10:260-265.

Kim JJ, Clark RE, Thompson RF (1995) Hippocampectomy impairs the memory of recently, but not remotely, acquired trace eyeblink conditioned responses. Behav Neurosci 109:195-203.

Kimble DP (1968) Hippocampus and internal inhibition. Psychol Bull 70:285-295.

Kishimoto Y, Kawahara S, Mori H, Mishina M, Kirino Y (2001) Long-trace interval eyeblink conditioning is impaired in mutant mice lacking the NMDA receptor subunit $\epsilon 1$. Eur J Neurosci 13:1221-1227.

Kishimoto Y, Fujimichi R, Araishi K, Kawahara S, Kano M, Aiba A, Kirino Y (2002) mGluR1 in cerebellar Purkinje cells is required for normal association of temporally contiguous stimuli in classical conditioning. Eur J Neurosci 16:2416-2424.

Levy WB (1996) A sequence predicting CA3 is a flexible associate that learns and uses context to solve hippocampus-like tasks. Hippocampus 6:579-590.

Levy WB, Steward O (1983) Temporal contiguity requirements for longterm associative potentiation/depression in the hippocampus. Neuroscience 8:791-797.

Lisman JE (1999) Relating hippocampal circuitry to function: recall of memory sequences by reciprocal dentate-CA3 interactions. Neuron 22:2333-2342.

Marr D (1971) Simple memory: a theory for archicortex. Philos Trans R Soc Lond B Biol Sci 262:23-81.

McEchron MD, Disterhoft JF (1999) Hippocampal encoding of non-spatial trace conditioning. Hippocampus 9:385-396.

McGlinchey-Berroth R, Carrillo MC, Gabrieli JD, Brawn CM, Disterhoft JF (1997) Impaired trace eye-blink conditioning in bilateral, medialtemporal lobe amnesia. Behav Neurosci 111:873-882.
Morris RG, McNaughton BL (1987) Hippocampal synaptic enhancement and information-storage within a distributed memory system. Trends Neurosci 10:408-415.

Moyer JRJ, Deyo RA, Disterhoft JF (1990) Hippocampectomy disrupts trace eye-blink conditioning in rabbits. Behav Neurosci 104:243-252.

Myers KM, Davis M (2002) Behavioral and neural analysis of extinction. Neuron 36:567-584.

Nakazawa K, Quirk MC, Chitwood RA, Watanabe M, Yeckel MF, Sun LD, Kato A, Carr CA, Johnston DJ, Wilson MA, Tonegawa S (2002) Requirement for hippocampal CA3 NMDA receptors in associative memory recall. Science 297:211-218.

Nakazawa K, Sun LD, Quirk MC, Rondi-Reig L, Wilson MA, Tonegawa S (2003) Hippocampal CA3 NMDA receptors are crucial for memory acquisition of one-time experience. Neuron 38:305-315.

Nakazawa K, McHugh TJ, Wilson MA, Tonegawa S (2004) NMDA receptors, place cells and hippocampal spatial memory. Nat Rev Neurosci 5:361-372.

Niki H (1966) Response preservation following hippocampal ablation in the rat. Jpn Psychol Res 8:1-9.

Pavlov IP (1927) Conditioned reflexes. London: Oxford UP.

Port RL, Romano AG, Steinmetz JE, Mikhail AA, Patterson MM (1986) Retention and acquisition of classical trace conditioned responses by rabbits with hippocampal lesions. Behav Neurosci 100:745-752.

Rodriguez P, Levy WB (2001) A model of hippocampal activity in trace conditioning: where's the trace? Behav Neurosci 115:1224-1238.

Sakamoto T, Takatsuki K, Kawahara S, Kirino Y, Niki H, Mishina M (2005) Role of hippocampal NMDA receptors in trace eyeblink conditioning. Brain Res 1039:130-136.

Solomon PR, Vander Schaaf EV, Thompson RF, Weisz DJ (1986) Hippocampus and trace conditioning of the rabbit's classically conditioned nictitating membrane response. Behav Neurosci 100:729-744.

Szapiro G, Vianna MR, McGaugh JL, Medina JH, Izquierdo I (2003) The role of NMDA glutamate receptors, PKA, MAPK, and CaMKII in the hippocampus in extinction of conditioned fear. Hippocampus 13:53-58.

Takatsuki K, Kawahara S, Takehara K, Kishimoto Y, Kirino Y (2001) Effects of the noncompetitive NMDA receptor antagonist MK-801 on classical eyeblink conditioning in mice. Neuropharmacology 41:618-628.

Thompson LT, Disterhoft JF (1997) $N$-methyl-D-aspartate receptors in associate eyeblink conditioning: both MK-801 and phencyclidine produce task- and dose-dependent impairments. J Pharmacol Exp Ther 281:928-940.

Thompson RF, Kim JJ (1996) Memory systems in the brain and localization of a memory. Proc Natl Acad Sci USA 93:13438-13444.

Thompson RF, Bao S, Chen L, Cipriano BD, Grethe JS, Kim JJ, Thompson JK, Tracy JA, Weninger MS, Krupa DJ (1997) Associative learning. San Diego: Academic.

Tseng W, Guan R, Disterhoft JF, Weiss C (2004) Trace eyeblink conditioning is hippocampally dependent in mice. Hippocampus 14:58-65.

Wallenstein GV, Eichenbaum H, Hasseimo ME (1998) The hippocampus as an associator of discontiguous events. Trends Neurosci 21:317-323.

Weiss C, Bouwmeester H, Power JM, Disterhoft JF (1999) Hippocampal lesions prevent trace eyeblink conditioning in the freely moving rat. Behav Brain Res 99:123-132.

Yeo C, Hesslow G (1998) Cerebellum and conditioned reflexes. Trends Cogn Sci 2:322-330. 